\title{
Exosomes derived from mangiferin-stimulated perivascular adipose tissue ameliorate endothelial dysfunction
}

\author{
QIANWEN ZHAO $^{1}$, JIE YANG $^{1}$, BAOLIN LIU $^{2}$, FANG HUANG $^{2}$ and YUEHUA LI ${ }^{1}$ \\ ${ }^{1}$ Key Laboratory of Targeted Intervention of Cardiovascular Disease, \\ Collaborative Innovation Center for Cardiovascular Disease Translational Medicine, \\ Nanjing Medical University, Nanjing, Jiangsu 211166; ${ }^{2}$ Department of Pharmacology of Chinese Materia Medic, \\ China Pharmaceutical University, Nanjing, Jiangsu 211198, P.R. China
}

Received October 17, 2018; Accepted March 20, 2019

DOI: $10.3892 / \mathrm{mmr} .2019 .10127$

\begin{abstract}
Perivascular adipose tissue (PVAT) is considered to serve a vital role during the development of endothelial dysfunction. The current study investigated the effect of exosomes derived from mangiferin-stimulated PVAT on endothelial function, including regeneration, migration, apoptosis and inflammation. The number of exosomes secreted by PVAT was increased by stimulation with mangiferin $(0.1,1$ or $10 \mu \mathrm{M})$, and uptake of these exosomes by endothelial cells was observed. Exosomes produced by stimulation of PVAT with mangiferin reversed the effects of inflammation-induced endothelial dysfunction following palmitic acid (PA) treatment. Furthermore, nuclear factor (NF)- $\kappa \mathrm{B}$ signaling in endothelial cells was significantly increased when treated with PA-induced PVAT-derived exosomes, whereas exosomes from the supernatant of PVAT stimulated with mangiferin reduced $\mathrm{p} 65$ and $\mathrm{p} 50$ phosphorylation levels in the cells, and inhibited p65 transportation to the nucleus. Taken together, the present study demonstrated that exosomes derived from mangiferin-stimulated PVAT supernatant inhibited inflammation-induced endothelial dysfunction via modulation of NF- $\mathrm{KB}$ signaling.
\end{abstract}

Correspondence to: Dr Yuehua Li, Key Laboratory of Targeted Intervention of Cardiovascular Disease, Collaborative Innovation Center for Cardiovascular Disease Translational Medicine, Nanjing Medical University, 818 Tianyuan Road, Nanjing, Jiangsu 211166, P.R. China

E-mail: yhli@njmu.edu.cn

Dr Fang Huang, Department of Pharmacology of Chinese Materia Medic, China Pharmaceutical University, 639 Longmian Road, Nanjing, Jiangsu 211198, P.R. China

E-mail: chengtianle007@163.com

Key words: mangiferin, perivascular adipose tissue, exosomes, endothelial dysfunction

\section{Introduction}

Adipose tissue has been recognized as a vital organ within the endocrine system, as it produces a wide range of hormones and cytokines, termed adipokines, which act in an autocrine or paracrine manner to regulate metabolic functions, inflammation, endothelial homeostasis and other processes (1). It is well established that obesity, particularly the increased prevalence of excessive visceral obesity, is closely associated with the rising incidence of cardiovascular diseases and type 2 diabetes $(2,3)$. One of the potential causes for the increasing risk is that adipose tissue dysfunction contributes directly and indirectly to vascular disease through the dysregulation of endothelial homeostasis (4). Recently, in an investigation of the interplay between adipose tissue and vascular function, attention was drawn to the regulation of endothelial homeostasis by perivascular adipose tissue (PVAT) (5). PVAT directly surrounds all blood vessels, thus factors secreted by adipocytes can easily target the vasculature. Although PVAT has characteristics similar to brown adipose tissue due to the generation of heat in the body (6), PVAT expands in obesity and diabetes, and is particularly susceptible to inflammation and loss of brown adipose tissue-like characteristics $(7,8)$. PVAT is different from subcutaneous adipose tissue and other visceral adipose tissue, and is therefore considered to be a distinct adipose tissue store, particularly regarding the regulation of vessel function (9).

Exosomes are nanovesicles, released into the extracellular space by various cell types, including adipose tissue (10). A previous study reported that exosomes contain substantial mRNAs, microRNAs and noncoding RNAs (11), which serve vital roles in regulating endothelial function, including migration, proliferation, apoptosis and angiogenesis (12-14). Furthermore, exosomes have an important role in paracrine processes, such as the crosstalk between endothelial cells and inflammatory cells (15). However, whether exosomes from PVAT regulate endothelial function has not been established to date.

Mangiferin is a xanthone glucoside present in Anemarrhena asphodeloides Bunge that is widely used in Chinese traditional medicine for the treatment of diabetes. Mangiferin exerts anti-inflammatory and anti-oxidant effects $(16,17)$, and these 
actions have been reported to be useful in the management of metabolic disorders $(18,19)$.

In the present study, exosomes released from PVAT were collected and used to stimulate endothelial cells in order to investigate the functional link between the endothelium and PVAT. The findings demonstrated that mangiferin regulated exosome release from PVAT and ameliorated endothelial dysfunction, involving nuclear factor- $\kappa \mathrm{B}(\mathrm{NF}-\kappa \mathrm{B})$. The current study increases the understanding on the effects of mangiferin in regulating adipose and endothelial function, which may be beneficial for its potential applications in the management of cardiovascular disorders associated with obesity.

\section{Materials and methods}

Animals. A total of 40 male Sprague-Dawley rats (8 weeks old, 180-200 g) were obtained from the Laboratory Animal Center of Nanjing Medical University (Nanjing, China). Animals were housed at a constant temperature $\left(22 \pm 1^{\circ} \mathrm{C}\right)$ in a 12:12-h light-dark cycle, with ad libitum access to a standard diet and water. The rats were cared for and treated in accordance with the Provisions and General Recommendation of Chinese Experimental Animals Administration Legislation. The present study received ethical approval from the Animal Ethics Committee of Nanjing Medical University.

Drugs and reagents. Mangiferin was purchased from Nanjing Zelang Medical Technological Co., Ltd. (Nanjing, China) and palmitic acid (PA) was from Sinopharm Chemical Reagent Co. Ltd. (Shanghai, China). The Annexin V-fluorescein isothiocyanate (FITC)/propidium iodide (PI) apoptosis detection kit (cat. no. KGA108) was obtained from Nanjing KeyGen Biotech Co., Ltd. (Nanjing, China). Matrigel was purchased from Corning, Inc. (Corning, NY, USA). Antibodies against phosphorylated

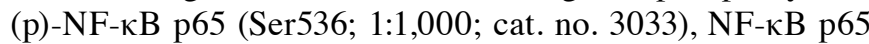
(1:1,000; cat. no. 8242), NF-кB p50 (1:1,000; cat. no. 32360), p-NF- $\kappa$ B p50 (1:1,000; cat. no. 28849), cluster of differentiation (CD)9 (1:1,000; cat. no. 9276), CD63 (1:1,000; cat. no. 59479) and $\beta$-tubulin (1:1,000; cat. no. 2146) were obtained from Cell Signaling Technology, Inc. (Danvers, MA, USA).

Endothelial cell isolation and culture. Rat arterial endothelial cells were isolated according to a standard protocol as previously described (20). Briefly, the rats were anesthetized with diethyl ether by inhalation prior to conducting the experiments. After the rats were sacrificed by decapitation following anesthesia, the arteries were excised and immersed in $75 \%$ ethanol for $30 \mathrm{sec}$ for disinfection. Next, the arteries were digested with 0.25 collagenase type II for $10 \mathrm{~min}$ and $0.25 \%$ trypsin for another $10 \mathrm{~min}$ at $37^{\circ} \mathrm{C}$. Cells were then centrifuged at $300 \mathrm{x}$ g for $6 \mathrm{~min}$ at $4^{\circ} \mathrm{C}$, resuspended in endothelial cell growth basal medium-2 (EBM-2; cat.no.cc-4176; Lonza Group, Ltd., Basel, Switzerland) with $10 \%$ fetal bovine serum (FBS; cat. no. 10100154, Gibco; Thermo Fisher Scientific, Inc., Waltham, MA, USA) and plated on laminin-coated dishes. Arterial endothelial cells at the third passage were used for further analyses.

Preparation of PVAT-derived exosomes. Rats were anesthetized with diethyl ether and sacrificed by decapitation. PVAT samples were obtained from the rat thoracic aorta, cut into small pieces, weighed to an equal size (50 mg) and rinsed using PBS. PVAT samples were cultured in Dulbecco's modified Eagle's medium (DMEM) supplemented with 10\% exosome-free FBS (cat. no. EXO-FBS500A-1; System Biosciences, Palo Alto, CA, USA). According to our previous study (21), the PVAT samples were cultured with PA $(100 \mu \mathrm{M})$ in the presence or absence of mangiferin $(0.1,1$ or $10 \mu \mathrm{M})$ for $2 \mathrm{~h}$; samples cultured without PA or mangiferin were used as the control group. Subsequent to washing with PBS, PVAT samples were incubated in DMEM for a further $22 \mathrm{~h}$. The supernatant was then collected as the conditioned medium (CM), and larger particles were removed by centrifugation at $10,000 \mathrm{x} \mathrm{g}$ for $5 \mathrm{~min}$ at $4^{\circ} \mathrm{C}$ and filtration through a $0.22-\mu \mathrm{m}$ filter. Exosomes in the PVAT CM were separated using total exosome isolation reagent (cat. no. 4478359; Invitrogen; Thermo Fisher Scientific, Inc.), and were observed by transmission electron microscopy (JEM-2000EX; JEOL, Ltd., Tokyo, Japan). Finally, exosomes derived from control, PA and PA + mangiferin groups were added to endothelial cells.

Immunofluorescence staining. Endothelial cells were seeded in 24-well plates, and then the cells were fixed with $4 \%$ paraformaldehyde for $30 \mathrm{~min}$ at $4^{\circ} \mathrm{C}$. Triton X-100 $(0.1 \%)$ was used to permeabilize endothelial cells prior to washing with PBS for three times. Endothelial cells were then blocked with $5 \%$ bovine serum albumin (BSA; Sangon Biotech Co., Ltd., Shanghai, China) for $20 \mathrm{~min}$ at room temperature. Cells were incubated overnight at $4^{\circ} \mathrm{C}$ with the primary antibodies (anti-CD63, anti-p65 and anti-p50) and then incubated at room temperature for $1 \mathrm{~h}$ with the secondary antibodies (Alexa Fluor ${ }^{\circledR}$ 594-conjugated donkey anti-rabbit secondary antibody; 1:100; cat. no. 1869587, Invitrogen; Thermo Fisher Scientific, Inc.). The cells were washed with PBS and the nuclei were stained with DAPI ( $20 \mu \mathrm{g} / \mathrm{ml}$; Thermo Fisher Scientific, Inc.) for $15 \mathrm{~min}$ at room temperature.

Preparation of thoracic aorta and assessment of endotheliumdependent relaxation. Rats were anesthetized with diethyl ether and sacrificed by decapitation. The thoracic aorta was stripped, immediately placed in $4^{\circ} \mathrm{C}$ Krebs-Henseleit $(\mathrm{K}-\mathrm{H})$ solution and incubated with $95 \% \mathrm{O}_{2}$ and $5 \% \mathrm{CO}_{2}$. Following careful removal of adherent tissues, four to eight samples of 3-mm long rings were cut. Subsequently, the aortic samples were suspended in an organ bath (SV-4; Chengdu Taimeng Software Co., Ltd., Chengdu, China) containing $10 \mathrm{ml} \mathrm{K-H}$ solution, and were maintained at $37^{\circ} \mathrm{C}$ and $\mathrm{pH} 7.4$, and continuously aerated with $95 \% \mathrm{O}_{2}$ and $5 \% \mathrm{CO}_{2}$. The aortic segments were exposed to $60 \mathrm{mM} \mathrm{KCl}$ to assess their functions following progressive stretching to a basal tension of $2.0 \mathrm{~g}$, and then stabilized for $\geq 90 \mathrm{~min}$. Exposure to $10 \mu \mathrm{M}$ acetylcholine (Sigma-Aldrich; Merck KGaA, Darmstadt, Germany) was used to relax the segment, which was pre-contracted with $1 \mu \mathrm{M}$ phenylephrine (Sigma-Aldrich; Merck KGaA). The integrity of the vascular endothelium was confirmed, while the diastolic rate reached $>80 \%$. The thoracic aorta was cultured with $\mathrm{CM}$ for $15 \mathrm{~min}$ in the organ bath, and the tone change was recorded and expressed in terms of tension.

Apoptosis analysis. Endothelial cells were incubated with exosomes obtained from the different treatment groups for $48 \mathrm{~h}$. 
Cells were subsequently collected using $0.25 \%$ trypsin without EDTA. Following three washes in PBS, cells were incubated with $500 \mu \mathrm{l}$ binding buffer containing $5 \mu \mathrm{l}$ Annexin V/FITC and $5 \mu \mathrm{l}$ PI (Nanjing KeyGen Biotech Co., Ltd.) for $30 \mathrm{~min}$ in the dark. Subsequently, apoptosis was measured using a BD FACSCalibur flow cytometer and data were analyzed using BD CellQuest ${ }^{\mathrm{TM}}$ (both from BD Biosciences, Franklin, Lakes, NJ, USA).

Transwell assay. Endothelial cells were incubated in EBM-2 at $37^{\circ} \mathrm{C}$ for 48 with exosomes $(10 \mu \mathrm{g} / \mathrm{ml})$ obtained from the different PVAT treatment groups. Cells were subsequently collected using $0.25 \%$ trypsin for $1 \mathrm{~min}$ at $37^{\circ} \mathrm{C}$ without EDTA seeded in the upper chambers 6-well Transwell inserts with an $8-\mu \mathrm{m}$ pore size at a density of $2 \times 10^{4}$ cells/well in normal EBM-2, and incubated at $37^{\circ} \mathrm{C}$ for $24 \mathrm{~h}$; normal EBM-2 with $10 \%$ FBS was added to the lower chambers. Non-migrated cells were gently removed with cotton swabs from the upper chamber, whereas cells that had migrated to the lower chamber were fixed using $4 \%$ paraformaldehyde at $4{ }^{\circ} \mathrm{C}$ for $24 \mathrm{~h}$ and stained with $0.1 \%$ crystal violet for $30 \mathrm{~min}$ at room temperature. The filters were washed with distilled water, and images were obtained using an inverted microscope (magnification, x100; BX41; Olympus Corporation, Tokyo, Japan).

Tube formation assay. Matrigel was thawed at $4^{\circ} \mathrm{C}$ for $24 \mathrm{~h}$ and then polymerized at $37^{\circ} \mathrm{C}$ for $1 \mathrm{~h}$. Following incubation of endothelial cells with exosomes derived from different PVAT groups for $48 \mathrm{~h}$ as aforementioned, cells were collected using $0.25 \%$ trypsin and resuspended to obtain a concentration of $2 \times 10^{5}$ cells $/ \mathrm{ml}$. Cells were then seeded on Matrigel-coated dishes for $8 \mathrm{~h}$. Tube formation was examined using a microscope (magnification, x200; IX51; Olympus Corporation).

ELISA. Endothelial cells were incubated with exosomes from different PVAT treatment groups for $48 \mathrm{~h}$ as aforementioned, and the supernatants were subsequently collected. Then, the supernatants were concentrated with ultrafiltration tubes (Amicon Ultra-3K; Merck KGaA) by centrifugation at $3,000 \mathrm{x} \mathrm{g}$ for $30 \mathrm{~min}$ at $4^{\circ} \mathrm{C}$. The concentrations of interleukin-6 (IL-6; cat. no. PR6000B) and tumor necrosis factor- $\alpha$ (TNF- $\alpha$; cat. no. PRTA00) were then determined using ELISA kits (R\&D Systems, Inc., Minneapolis, MN, USA).

Reverse transcription-quantitative polymerase chain reaction $(R T-q P C R)$ analysis. Endothelial cells were incubated with exosomes obtained from PVAT treated with PA and mangiferin for $24 \mathrm{~h}$ as aforementioned. Total RNA was extracted using TRIzol ${ }^{\circledR}$ reagent (Thermo Fisher Scientific, Inc.) according to the manufacturer's protocol. The extracted RNA was dissolved in diethyl pyrocarbonate (DEPC)-treated water, and the RNA concentration was determined by optical density measurement at $260 \mathrm{~nm}$ using a spectrophotometer. RT was subsequently performed on RNA samples $(2 \mu \mathrm{g})$ using the PrimeScript ${ }^{\mathrm{TM}}$ RT reagent (Takara Bio, Inc., Otsu, Japan), following the manufacturer's protocol. cDNA (1 $\mu \mathrm{l})$ mixed with Ssofast ${ }^{\mathrm{TM}}$ EvaGreen $^{\circledR}$ Supermix (Bio-Rad Laboratories, Inc., Hercules, CA, USA), DEPC-treated water, forward primer and reverse primer (Sangon Biotech Co., Ltd.) to obtain a 20- $\mu 1$ reaction mixture was used for qPCR (22). qPCR was conducted as follows: $95^{\circ} \mathrm{C}$ for $30 \mathrm{sec}$, then 40 cycles of $95^{\circ} \mathrm{C}$ for $5 \mathrm{sec}$ and $60^{\circ} \mathrm{C}$ for $30 \mathrm{sec}$. The primers used in qPCR are presented in Table I. Expression was quantified using the $2^{-\Delta \Delta \mathrm{Cq}}$ method and normalized to the endogenous control $\beta$-actin (23).

Detection of intracellular nitric oxide (NO). Cells were cultured in 6-well plates and treated with exosomes from the various groups as aforementioned upon reaching $70 \%$ confluence. The secretion of $\mathrm{NO}$ was determined $48 \mathrm{~h}$ later using an NO test kit (cat. no. A013-2, Nanjing Jiancheng Bioengineering Institute Co., Ltd., Nanjing, China) according to the manufacturer's protocol.

Western blot analysis. Briefly, the total protein was extracted from the samples using radioimmunoprecipitation assay lysis buffer (Beyotime Institute of Biotechnology, Shanghai, China). Protein concentrations were quantified using a Bicinchoninic Acid Protein Assay kit (Biosky Biotechnology, Nanjing, China) according to the manufacturer's protocol. Next, protein samples (30 $\mu \mathrm{g} /$ lane) were separated by SDS-PAGE on $10 \%$ gels and transferred to polyvinylidene difluoride membranes $(0.45 \mu \mathrm{m}$; EMD Millipore, Billerica, MA, USA). Membranes were blocked for $2 \mathrm{~h}$ at room temperature with 1-5\% BSA (Sangon Biotech Co., Ltd.) in TBS-Tween-20 [containing $5 \mathrm{mmol} / 1$ Tris- $\mathrm{HCl}$ (pH 7.6), $136 \mathrm{mmol} / \mathrm{l} \mathrm{NaCl}$ and $0.05 \%$ Tween-20] and then incubated with the aforementioned antibodies against p65, p-p65, p50, p-p50, CD9, CD63 and tubulin overnight at $4^{\circ} \mathrm{C}$. Subsequently, the membranes were incubated with secondary antibody [horseradish peroxidase-conjugated anti-rabbit immunoglobulin G (IgG; cat. no. A0208) or anti-mouse IgG (cat. no. A0216); 1:5,000; Beyotime Institute of Biotechnology] at $37^{\circ} \mathrm{C}$ for $2 \mathrm{~h}$. Signals were detected using enhanced chemiluminescence western detection reagents (Thermo Fisher Scientific, Inc.) and quantified using Image-Lab version 5.2.1 software (Bio-Rad Laboratories, Inc.).

Statistical analysis. All values are presented as the mean \pm standard deviation. The statistical significance between mean values was evaluated using one-way analysis of variance, followed by Tukey's test. Statistical analyses were performed using SPSS software, version 23.0 (IBM Corp., Armonk, NY, USA). P<0.05 was considered to indicate a statistically significant difference.

\section{Results}

Mangiferin promotes the release of exosomes from PVAT. Initially, the effect of mangiferin on the release of exosomes was determined. As demonstrated in Fig. 1A, a large number of exosomes were present in the CM supernatant. Exosomes were extracted and concentrated from PVAT CM following stimulation with PA and mangiferin $(0.1,1$ and $10 \mu \mathrm{M})$, or control. PA evidently inhibited the release of exosomes from PVAT compared with the control group. However, the number of PVAT-released exosomes was markedly increased by mangiferin under PA-induced inflammation conditions in a dose-dependent manner. Subsequently, exosomes were extracted from $\mathrm{CM}$ using total exosome isolation reagents. A total of $10 \mu \mathrm{g}$ exosomes was selected in all groups to ensure that the same concentration of exosomes was added to the 
Table I. Primers of TNF- $\alpha$, IL-6, ARG1 and $\beta$-actin.

\begin{tabular}{lll}
\hline Gene & \multicolumn{1}{c}{ Forward primer $\left(5^{\prime}-3^{\prime}\right)$} & \multicolumn{1}{c}{ Reverse primer (5'-3') } \\
\hline TNF- $\alpha$ & GAGTAAGGGGATGCAGCTAAGA & CAGTTTCAGGGCAAGAAGTACC \\
IL-6 & TCTTGGGACTGATGTTGTTGAC & GGGTGGTATCCTCTGTGAAGTC \\
ARG1 & ATCATGGAAGTGAACCCACTC & TCCAAAACAAGACAAGGTCAC \\
$\beta$-actin & GACGTTGACATCCGTAAAGACC & TGCTAGGAGCCAGGGCAGTA
\end{tabular}

TNF- $\alpha$, tumor necrosis factor- $\alpha$; IL-6, interleukin-6; ARG1, arginase-1.

endothelial cell culture. As displayed in Fig. 1B, the results revealed that the expression of $\mathrm{CD} 9$ and $\mathrm{CD} 63$ proteins, which are specific markers of exosomes, was similar in all groups. The uptake of exosomes by endothelial cells was similar when $\mathrm{CM}$ that was obtained from PVAT stimulated with mangiferin and PA was used, indicating that the intake of exosomes by endothelial cells was not influenced by the pre-treatment with mangiferin or PA. Immunofluorescence further demonstrated the uptake of PVAT-derived exosomes by endothelial cells (Fig. 1C).

Mangiferin-induced PVAT-derived exosomes regulate endothelial function. Further experiments were performed to determine whether exosomes from mangiferin-stimulated PVAT influence the endothelial function. Preliminary results indicated that the effect of exosomes on endothelial cells in vitro was not altered following 24-h exposure of the cells to exosomes (data not shown). Thus, 48-h exposure of endothelial cells to exosomes was conducted in the current study in order to observe the regeneration and apoptosis levels. Exosomes produced following mangiferin treatment $(1$ and $10 \mu \mathrm{M})$ markedly promoted the tube formation ability of endothelial cells compared with the PA-induced exosomes (Fig. 2A). In addition, the migration of endothelial cells was inhibited by PA-induced exosomes, whereas migration was clearly increased by mangiferin $(10 \mu \mathrm{M})$-induced exosomes compared with PA-induced exosomes (Fig. 2B). The apoptosis level was also determined in these treatment groups, and the data revealed that mangiferin (1 and $10 \mu \mathrm{M})$-induced exosomes significantly reduced the apoptosis of endothelial cells compared with PA-induced exosomes (Fig. 2C). Organ bath analysis identified that PA-induced PVAT-derived exosomes significantly stimulated the constriction of normal aortic rings compared with the control, whereas mangiferin ( 1 and $10 \mu \mathrm{M})$-induced exosomes significantly promoted the relaxation of aortic rings compared with PA-induced exosomes (Fig. 2D), indicating that the relaxation of aortic rings was blocked by exosomes produced from PA-stimulated PVAT. However, this effect was reversed upon the use of exosomes produced by mangiferin-treated PVAT, ameliorating the endothelial dysfunction caused by PA-induced adipose tissue inflammation.

Mangiferin-induced PVAT-derived exosomes inhibit endothelial inflammation. Endothelial inflammation induced by PA treatment was further analyzed using RT-qPCR and ELISA. The mRNA expression levels of the inflammatory factors IL- 6 and TNF- $\alpha$ were significantly increased in endothelial cells treated with PA-induced PVAT-derived exosomes (PA alone group) compared with the control group (Fig. 3A); however, these effects were significantly reversed in the cells incubated with mangiferin-induced exosomes. In addition, the levels of ARG1 mRNA in endothelial cells were significantly upregulated upon incubation with mangiferin-induced PVAT-derived exosomes compared with PA-induced exosomes (Fig. 3A). As compared with the PA-stimulated alone group, exosomes produced by mangiferin-stimulated PVAT $(0.1,1$ and $10 \mu \mathrm{M})$ significantly inhibited the release of IL-6 and TNF- $\alpha$ proteins (Fig. 3B), and promoted $\mathrm{NO}$ generation in endothelial cells (Fig. 3C). These results demonstrated that exosomes from mangiferin-stimulated PVAT reduced endothelial inflammation and increased endothelial relaxation.

Mangiferin-induced PVAT-derived exosomes block NF- $\kappa B$ signaling. As mangiferin-induced PVAT-derived exosomes ameliorated endothelial inflammation, the mechanisms underlying the effect of mangiferin-induced exosomes on endothelial cells were investigated. Mangiferin $(10 \mu \mathrm{M})$-induced PVAT-derived exosomes significantly inhibited p65 phosphorylation in endothelial cells compared with the effect of PA-induced exosomes (Fig. 4A). Furthermore, mangiferin $(10 \mu \mathrm{M})$-induced PVAT-derived exosomes also markedly reduced p50 phosphorylation in endothelial cells (Fig. 4B). The translocation of p65 and p50 in endothelial cells treated with PVAT-derived exosomes was also detected. PA-induced PVAT-derived exosomes evidently promoted the translocation of p65 and p50, whereas the effects were reversed when exosomes from mangiferin $(10 \mu \mathrm{M})$-treated PVAT were used (Fig. 4C and D). These results demonstrated that exosomes produced from mangiferin-treated PVAT reduced the activation of NF- $\kappa \mathrm{B}$ signaling.

\section{Discussion}

It is well established that adipose tissue inflammation-induced cardiovascular diseases are associated with diabetes and obesity. In particular, PVAT dysfunction serves a vital role in endothelial injury, which may result in vascular damage (24). Additionally, the anatomical and functional features of PVAT $d$ etermine its vital role in the regulation of endothelial homeostasis (25). PVAT inflammation is known to be involved in the regulation of endothelial function via inhibition of AMP-activated protein kinase (AMPK) signaling, and mangiferin has been reported to ameliorate endothelial injury by reducing PVAT 
A

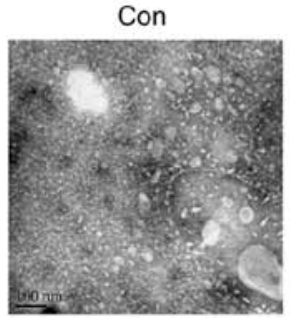

B
PA

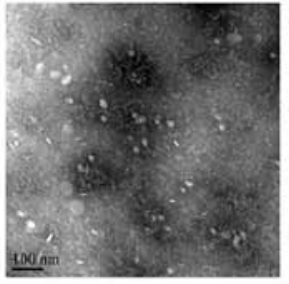

$0.1 \mu \mathrm{M}+\mathrm{PA}$

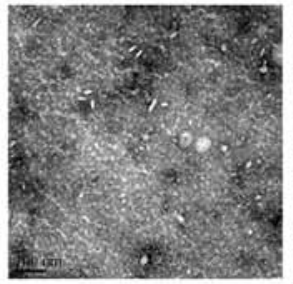

$1 \mu \mathrm{M}+\mathrm{PA}$

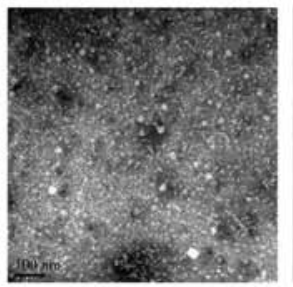
Mangiferin
0.1
1
$10 \mu \mathrm{M}$

CD9

CD63

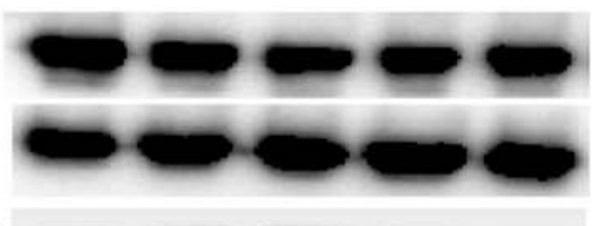

Tubulin

C
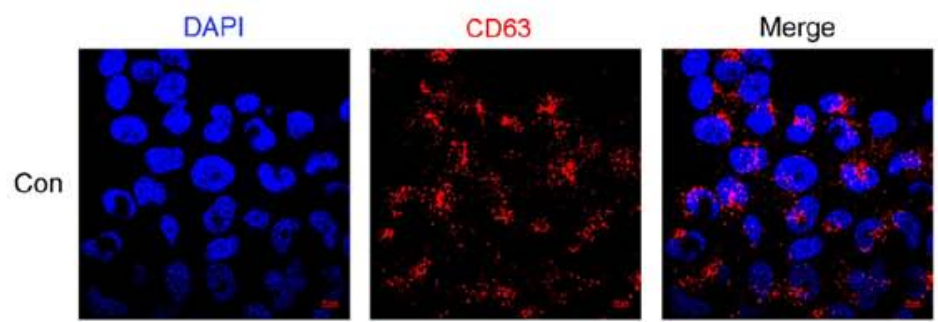

PA
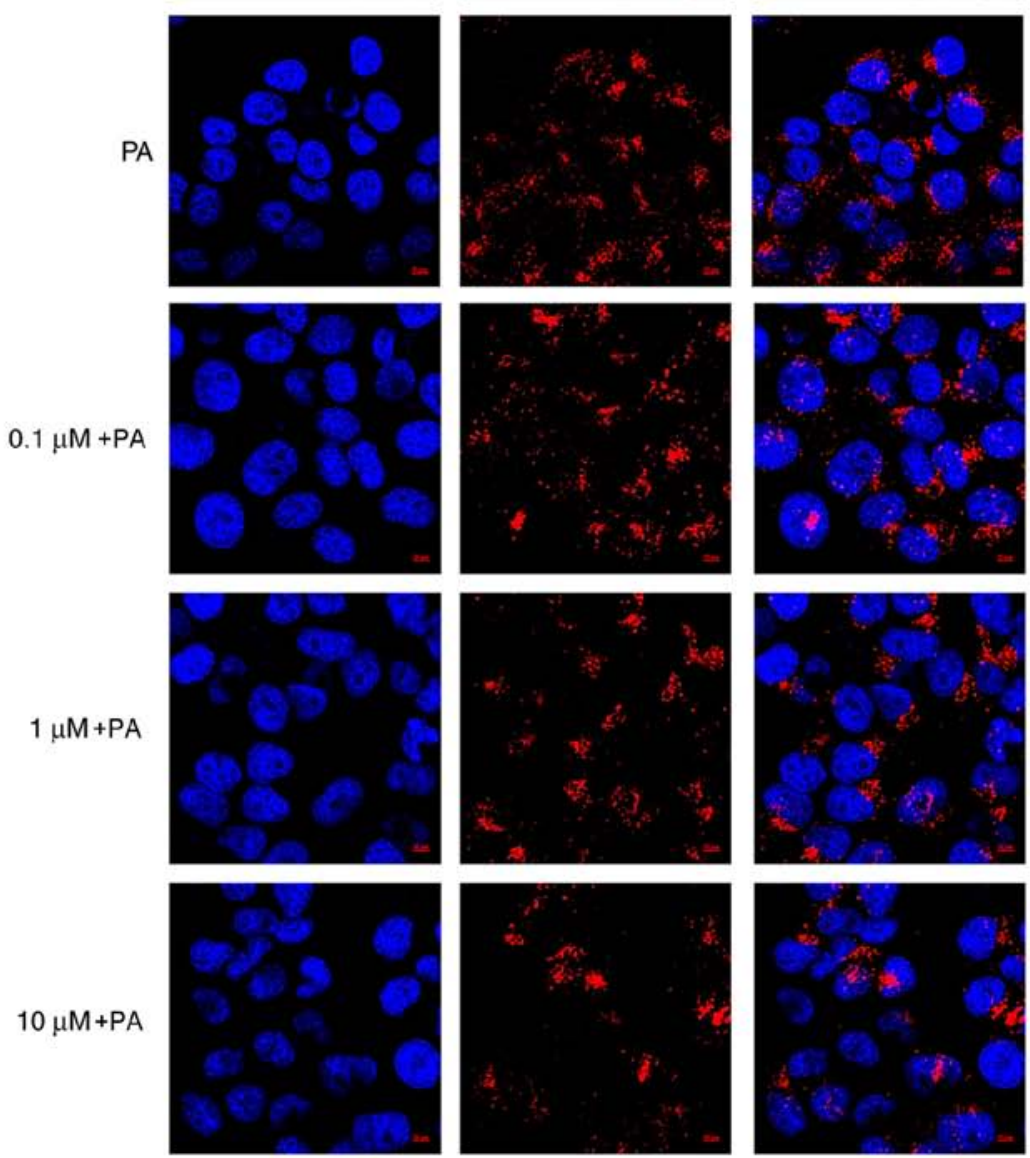

Figure 1. Mangiferin promotes the release of exosomes from PVAT. PVAT was collected from normal rats and cultured with PA (100 $\mu \mathrm{M})$ in the presence or absence of mangiferin $(0.1,1$ or $10 \mu \mathrm{M})$ for $2 \mathrm{~h}$; samples cultured without PA and mangiferin were used as control groups. Subsequent to washing with PBS, PVAT was incubated in fresh DMEM for a further $22 \mathrm{~h}$. Conditioned medium was collected from all treatment groups, and exosomes were extracted from this medium using a total exosome isolation reagent. (A) Transmission electron microscopy was used to observe the release of exosomes from PVAT with or without PA and mangiferin stimulation (magnification, x100,000). (B) CD9 and CD63 protein expression of different groups, examined by western blot assay. (C) Exosomes were extracted from the supernatants of PVAT, and then immunofluorescence was used to observe the ingestion of exosomes in endothelial cells. (magnification, x630). Results are representative of three independent experiments. PVAT, perivascular adipose tissue; PA, palmitic acid; Con, Control. 
A

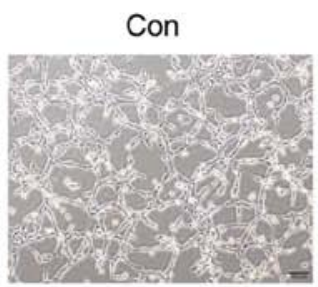

B

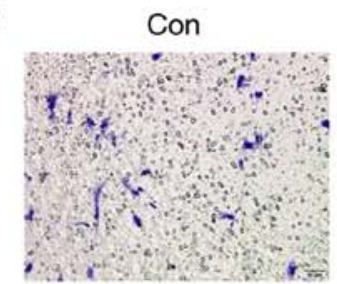

PA

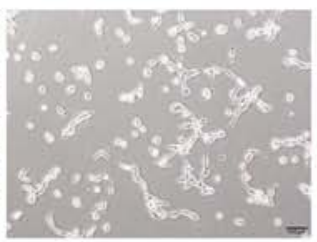

PA
$0.1 \mu \mathrm{M}+\mathrm{PA}$

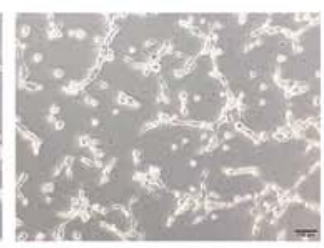

$1 \mu \mathrm{M}+\mathrm{PA}$

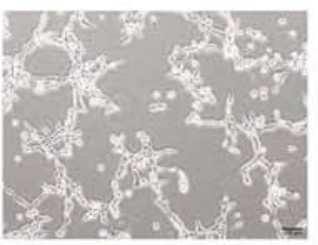

$10 \mu \mathrm{M}+\mathrm{PA}$

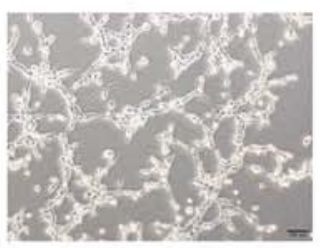

$0.1 \mu \mathrm{M}+\mathrm{PA}$

$1 \mu \mathrm{M}+\mathrm{PA}$

$10 \mu \mathrm{M}+\mathrm{PA}$
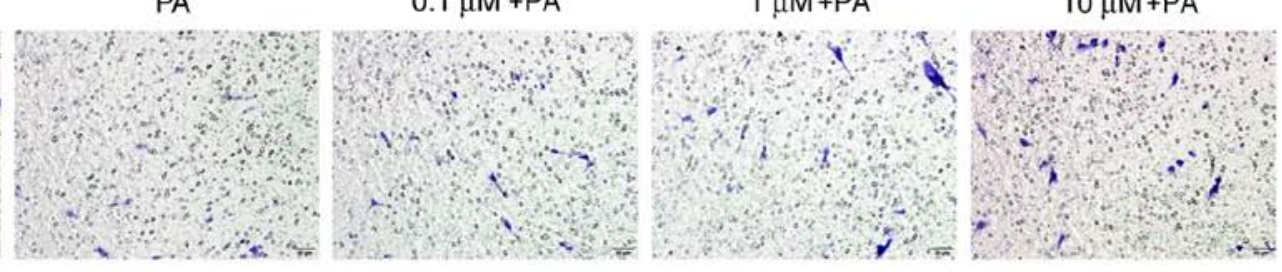

C
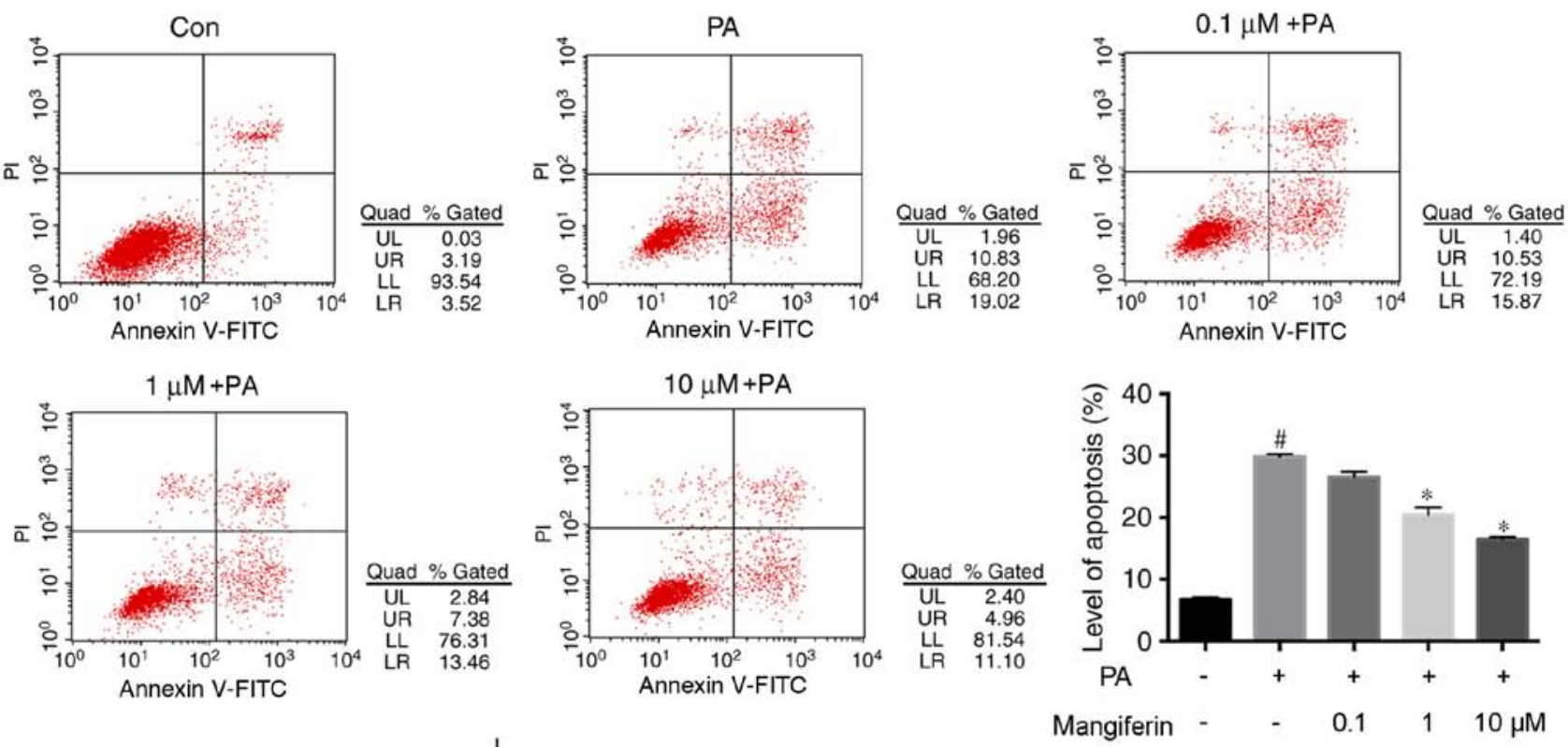

D
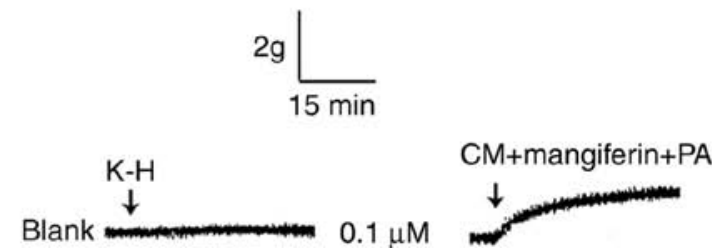

$0.1 \mu \mathrm{M}$

$\mathrm{CM}+$ mangiferin+PA
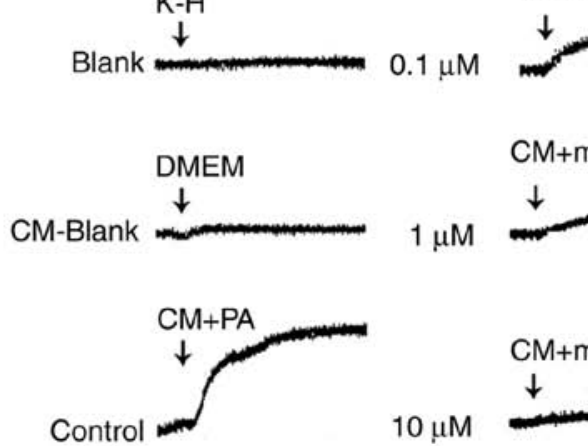

$\mathrm{CM}+$ mangiferin+PA
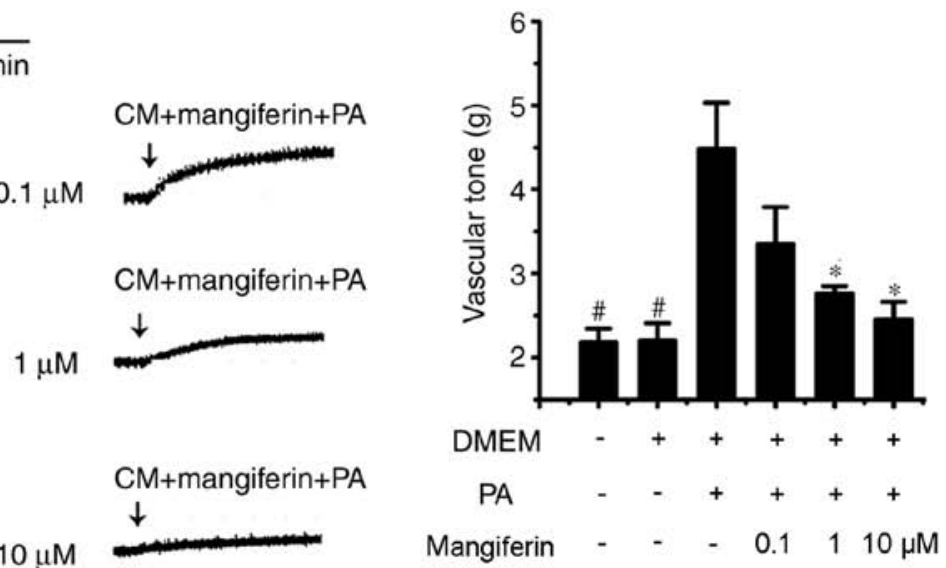

Figure 2. Exosomes from PVAT regulate endothelial function. Endothelial cells were treated with exosomes derived from PVAT, which was pre-treated with or without mangiferin $(0.1,1$ or $10 \mu \mathrm{M})$ and PA $(100 \mu \mathrm{M})$ for $2 \mathrm{~h}$, and then incubated without mangiferin and PA for a further $22 \mathrm{~h}$. (A) Angiogenesis was detected by tube formation experiments (magnification, x200). (B) Endothelial cell migration was detected by conducting a transwell assay (magnification, x100). (C) Flow cytometry analysis was used to evaluate the level of apoptosis. (D) Organ bath analysis of the relaxation of normal aortic rings incubated with exosomes pretreated with or without PA and mangiferin. Results are representative of three independent experiments. ${ }^{\#} \mathrm{P}<0.05$ vs. control group; ${ }^{\mathrm{P}}<0.05$ vs. $\mathrm{PA}$ group. PVAT, perivascular adipose tissue; PA, palmitic acid; CM, conditioned medium; DMEM, Dulbecco's modified Eagle's medium; K-H, Krebs-Henseleit; Con, Control.

inflammation (26); however, the major regulatory mechanisms are not clearly understood. The aim of the current study was to research the potential mechanism by which mangiferin regulates PVAT inflammation-induced endothelial dysfunction. 

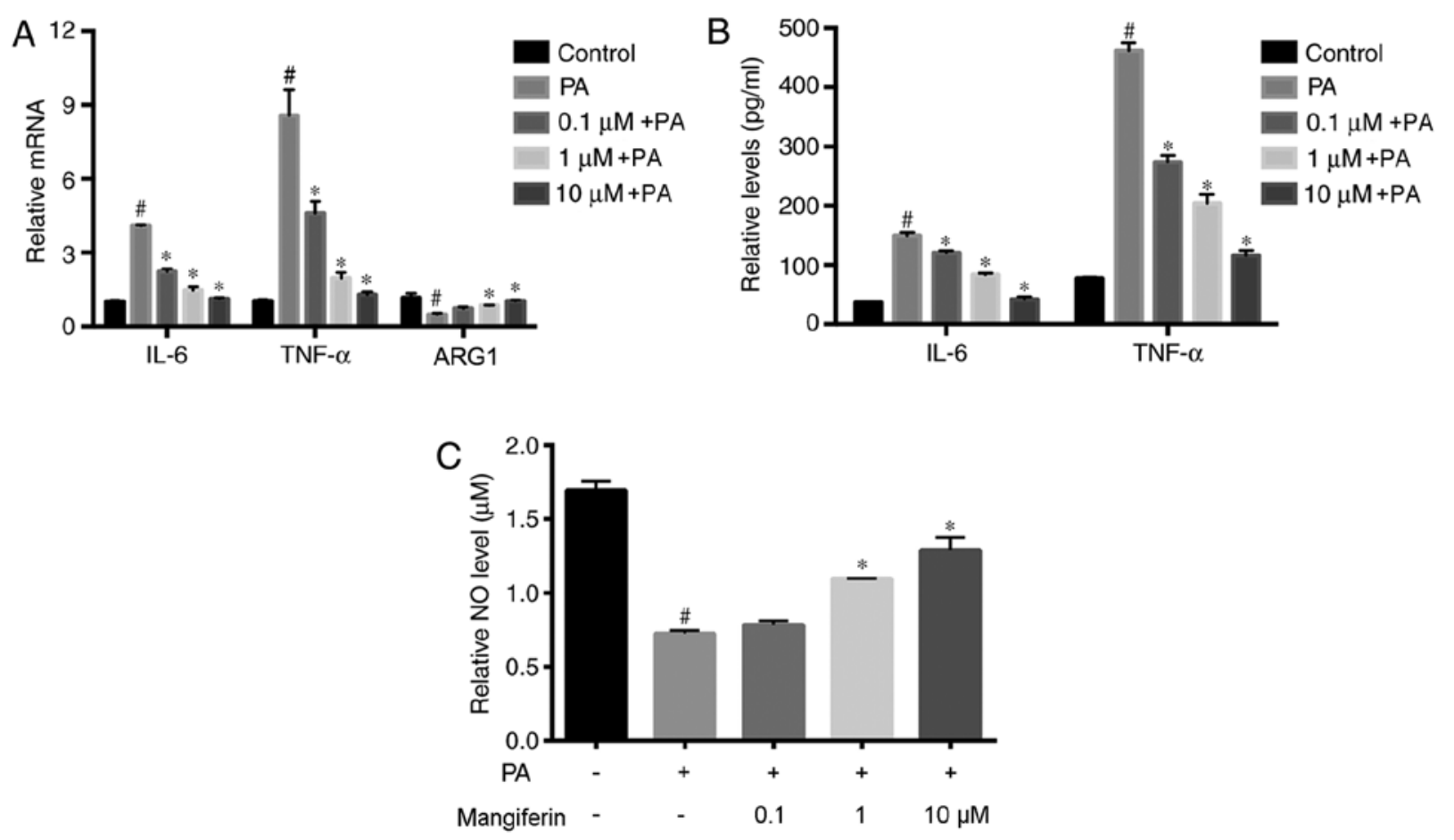

Figure 3. Exosomes derived from PVAT inhibit endothelial inflammation. Endothelial cells were treated with exosomes from PVAT, which had been pre-treated with or without mangiferin $(0.1,1$ or $10 \mu \mathrm{M})$ and PA $(100 \mu \mathrm{M})$ for $2 \mathrm{~h}$, and then incubated without mangiferin and PA for a further $22 \mathrm{~h}$. (A) Relative mRNA expression levels of IL-6, ARG1 and TNF- $\alpha$ were detected by reverse transcription-quantitative polymerase chain reaction. (B) Content of IL-6 and TNF- $\alpha$ in endothelial cells and (C) NO released from endothelial cells were detected by ELISA. Results are representative of six independent experiments. ${ }^{\#} \mathrm{P}<0.05$ vs. control group; ${ }^{*} \mathrm{P}<0.05$ vs. PA group. PVAT, perivascular adipose tissue; PA, palmitic acid; IL-6, interleukin-6; ARG1, arginase-1; TNF- $\alpha$, tumor necrosis factor- $\alpha$; NO, nitric oxide.

In obesity, adipose tissue dysfunction commonly causes increased release of free fatty acids (FFAs), which elevating their level in circulation (27). Since PA is a major component of the total serum FFAs, PVAT was stimulated with PA to induce endothelial injury in the present study. As previous research identified that PA-induced PVAT inflammation promotes endothelial dysfunction (28), CM was collected following treatment of PVAT with PA and mangiferin. Saez et al (29) has reported that extracellular vesicles (EVs), including exosomes $(50-100 \mathrm{~nm})$, contribute to defective insulin sensitivity in the vasculature, which has a vital role in cardiovascular complications (27). Furthermore, EVs are considered to be important mediators of endocrine functions through cell to cell communication. Exosomes can transfer Delta-like 4 (which is a Notch ligand) between endothelial cells, resulting in inhibition of angiogenesis through Notch signaling (30). Thus, in the current study, exosomes were isolated from $\mathrm{CM}$ following stimulation of PVAT with PA, PA + mangiferin and control groups. PA stimulation reduced the release of exosomes; by contrast, mangiferin increased exosome release from PVAT, even when combined with PA. Furthermore, it was confirmed that the exosomes released from PVAT stimulated with PA and mangiferin were taken up by endothelial cells, suggesting that mangiferin stimulation of PVAT can have paracrine effects on endothelial cells via exosomes.

Our previous studies have demonstrated that mangiferin-containing polyphenols of Anemarrhena asphodeloides inhibit inflammation in adipocytes, and marginally in endothelial cells, via regulation of the AMPK pathway $(21,31)$; thus, it would be of interest to determine whether mangiferin induces indirect effects on endothelial cells. It has been reported that endothelial dysfunction is associated with cell apoptosis (32). In addition, PA has previously been demonstrated to induce inflammation and apoptosis in human umbilical vein endothelial cells by regulating oxidative and endoplasmic reticulum stress (33). Adipose-derived stem cells have been investigated for their use as potential pro-angiogenic therapeutics tools (34), suggesting that angiogenesis is closely associated with adipose-derived factors. Consistent with this, the present study observed that exosomes derived from PA-stimulated PVAT induced endothelial dysfunction, which was indicated by features including reduced cell regeneration, reduced cell migration, increased cell apoptosis and inhibition of vessel dilation. However, PVAT treatment with mangiferin promoted the release of exosomes and further ameliorated the PA-induced PVAT inflammation-associated endothelial injury.

PA evokes inflammation with dysregulation of adipokine expression in PVAT, as demonstrated by enhanced NF- $\mathrm{KB}$ p65 phosphorylation, increased expression of pro-inflammatory adipokines and reduced expression of ARG1 (35). The current study demonstrated that exosomes produced by PA-stimulated PVAT induced the expression of pro-inflammatory factors (IL-6 and TNF- $\alpha$ ) in endothelial cells. Furthermore, endothelial injury is known to be closely associated with the activation of NF- $\kappa$ B signaling (36). The findings of the current study revealed that PA-induced PVAT inflammation promoted NF- $\mathrm{\kappa B}$ activation in endothelial cells, as indicated by increased p65 and p50 phosphorylation levels and protein translocation into the nucleus. However, treatment of PVAT with mangiferin 
$\mathrm{A}_{\mathrm{PA}} \mathrm{PA}_{+}++$

B
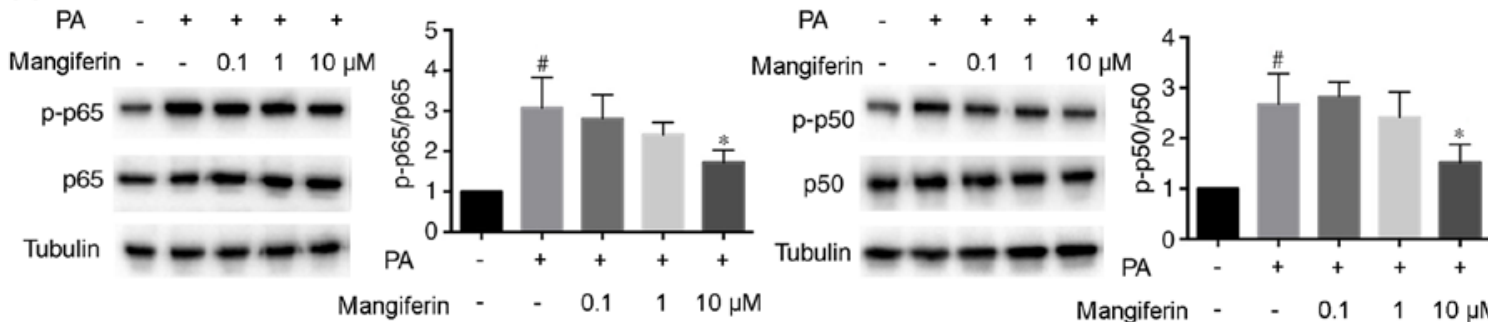

C

DAPI
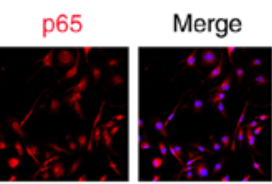

D

Mangiferin

$0.1 \quad 1 \quad 10 \mu \mathrm{M}$
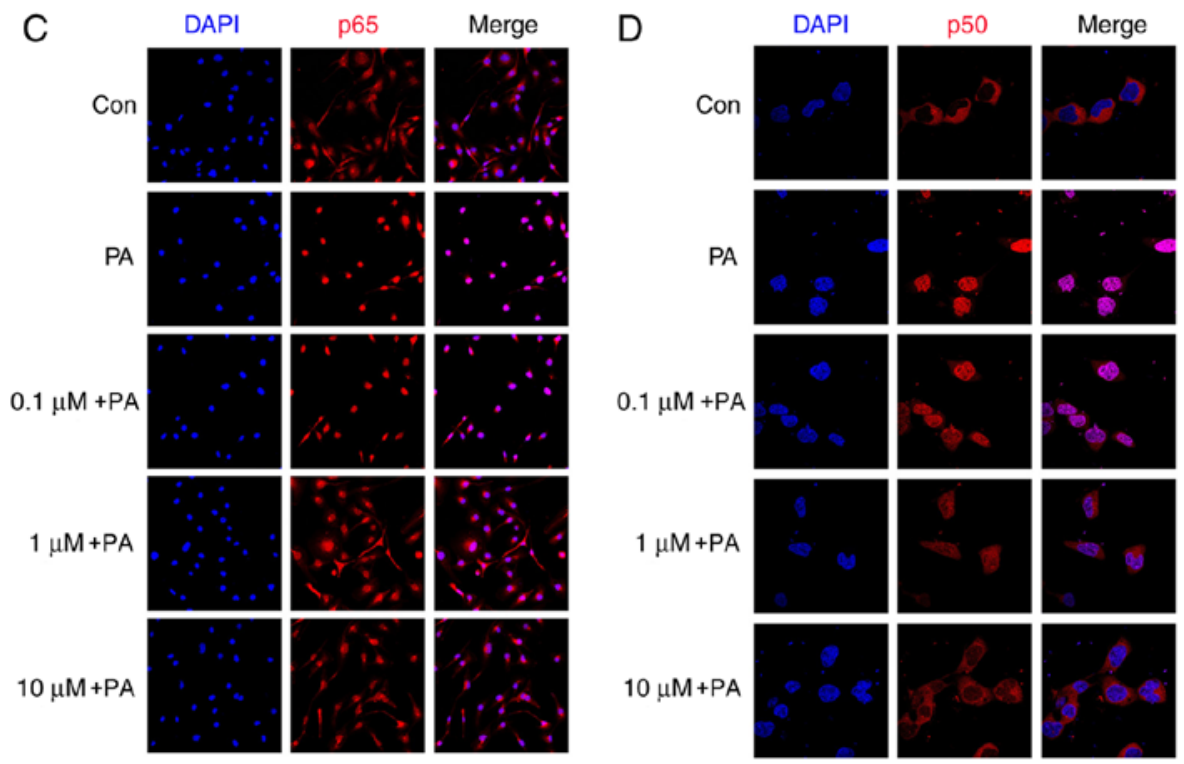

Figure 4. Mangiferin-stimulated exosomes derived from PVAT inhibit the activation of nuclear factor- $\mathrm{kB}$ signaling in endothelial cells by decreasing p65 and p50 expression through. Endothelial cells were treated with exosomes from PVAT, which was preprocessed with or without mangiferin $(0.1,1$ or $10 \mu \mathrm{M})$ and PA $(100 \mu \mathrm{M})$ for $2 \mathrm{~h}$, and then incubated without mangiferin and PA for a further $22 \mathrm{~h}$. (A) Western blot analysis identified the levels of (A) p65 phosphorylation and (B) p50 phosphorylation. (C) p65 (magnification, x400) and (D) p50 (magnification, x630) translocation was detected by immunofluorescence in endothelial cells with or without PVAT-derived exosomes that were stimulated with or without mangiferin. Results are representative of three independent experiments. ${ }^{\#} \mathrm{P}<0.05$ vs. control group; ${ }^{*} \mathrm{P}<0.05$ vs. PA group. PVAT, perivascular adipose tissue; $\mathrm{PA}$, palmitic acid.

promoted the release of exosomes and ameliorated the endothelial inflammation caused by PA stimulation of PVAT.

In conclusion, it is recognized that adipose tissue exerts profound effects on vascular function via secretion of various bioactive molecules. In the current study, exosomes were extracted from CM following the stimulation of PVAT with PA and mangiferin. Endothelial cells were then incubated with the PVAT-derived exosomes to determine the indirect effects of mangiferin on the endothelial function via regulation of PVAT function. The results revealed that PVAT-derived exosomes induced endothelial dysfunction, and that exosomes derived from PVAT treated with mangiferin promoted angiogenesis and migration, and inhibited apoptosis in endothelial cells. Taken together, mangiferin was demonstrated to ameliorate endothelial dysfunction via exosomes derived from PA-stimulated PVAT.

\section{Acknowledgements}

Not applicable.

\section{Funding}

This study was funded by the Major Research Plan of the National Natural Science Foundation of China (grant no. 91639115).

\section{Availability of data and materials}

The datasets used and analyzed during the current study are available from the corresponding author on reasonable request.

\section{Authors' contributions}

YL and FH designed the study and interpreted the data. QZ wrote the manuscript and performed the experiments. JY and $\mathrm{BL}$ analyzed the data and were involved in drafting the manuscript. All authors read and approved the final manuscript.

\section{Ethics approval and consent to participate}

This research was approved by the Animal Ethics Committee of Nanjing Medical University (Nanjing, China).

\section{Patient consent for publication}

Not applicable.

\section{Competing interests}

The authors declare that they have no competing interests. 


\section{References}

1. Bassols J, Ortega FJ, Moreno-Navarrete JM, Peral B, Ricart W and Fernández-Real JM: Study of the proinflammatory role of human differentiated omental adipocytes. J Cell Biochem 107: 1107-1117, 2009

2. Goldberg RB: Cytokine and cytokine-like inflammation markers, endothelial dysfunction, and imbalanced coagulation in development of diabetes and its complications. J Clin Endocrinol Metab 94: 3171-3182, 2009

3. Mokdad AH, Ford ES, Bowman BA, Dietz WH, Vinicor F, Bales VS and Marks JS: Prevalence of obesity, diabetes, and obesity-related health risk factors. JAMA 289: 76-79, 2003.

4. Sun X, Lin J, Zhang Y, Kang S, Belkin N, Wara AK, Icli B, Hamburg NM, Li D and Feinberg MW: MicroRNA-181b improves glucose homeostasis and insulin sensitivity by regulating endothelial function in white adipose tissue. Circ Res 118: 810-821, 2016.

5. Schinzari F, Tesauro $M$ and Cardillo C: Endothelial and perivascular adipose tissue abnormalities in obesity-related vascular dysfunction: Novel targets for treatment. J Cardiovasc Pharmacol 69: 360-368, 2017.

6. Fitzgibbons TP, Kogan S, Aouadi M, Hendricks GM, Straubhaar J and Czech MP: Similarity of mouse perivascular and brown adipose tissues and their resistance to diet-induced inflammation. Am J Physiol Heart Circ Physiol 301: H1425-H1437, 2011.

7. Braunersreuther V, Mach $F$ and Steffens S: The specific role of chemokines in atherosclerosis. Thromb Haemost 97: 714-721, 2007.

8. Verhagen SN and Visseren FL: Perivascular adipose tissue as a cause of atherosclerosis. Atherosclerosis 214: 3-10, 2011.

9. Brown NK, Zhou Z, Zhang J, Zeng R, Wu J, Eitzman DT, Chen YE and Chang L: Perivascular adipose tissue in vascular function and disease: A review of current research and animal models. Arteriscler Thromb Vasc Biol 34: 1621-1630, 2014.

10. Zhao H, Shang Q, Pan Z, Bai Y, Li Z, Zhang H, Zhang Q, Guo C, Zhang L and Wang Q: Exosomes from adipose-derived stem cells attenuate adipose inflammation and obesity through polarizing M2 macrophages and beiging in white adipose tissue. Diabetes 67: 235-247,2018.

11. Kowal J, Tkach M and Thery C: Biogenesis and secretion of exosomes. Curr Opin Cell Biol 29: 116-125, 2014.

12. Lee HD, Kim YH and Kim DS: Exosomes derived from human macrophages suppress endothelial cell migration by controlling integrin trafficking. Eur J Immunol 44: 1156-1169, 2014.

13. Wu XM, Gao YB, Cui FQ and Zhang N: Exosomes from high glucose-treated glomerular endothelial cells activate mesangial cells to promote renal fibrosis. Biol Open 5: 484-491, 2016.

14. Castelli G, Parolini I, Cerio AM, D'Angiò A, Pasquini L, Carollo M, Sargiacomo M, Testa U and Pelosi E: Conditioned medium from human umbilical vein endothelial cells markedly improves the proliferation and differentiation of circulating endothelial progenitors. Blood Cells Mol Dis 61: 58-65, 2016.

15. Raposo G and Stoorvogel W: Extracellular vesicles: Exosomes, microvesicles, and friends. J Cell Biol 200: 373-383, 2013.

16. Garrido G, González D, Lemus Y, García D, Lodeiro L, Quintero G, Delporte C, Núñez-Sellés AJ and Delgado R: In vivo and in vitro anti-inflammatory activity of Mangifera indica $L$. extract (VIMANG). Pharmacol Res 50: 143-149, 2004.

17. Sánchez GM, Re L, Giuliani A, Núñez-Sellés AJ, Davison GP and León-Fernández OS: Protective effects of Mangifera indica $L$. extract, mangiferin and selected antioxidants against TPA-induced biomolecules oxidation and peritoneal macrophage activation in mice. Pharmacol Res 42: 565-573, 2000.

18. Guo F, Huang C, Liao X, Wang Y, He Y, Feng R, Li Y and Sun C: Beneficial effects of mangiferin on hyperlipidemia in high-fat-fed hamsters. Mol Nutr Food Res 55: 1809-1818, 2011.

19. Niu Y, Li S, Na L, Feng R, Liu L, Li Y and Sun C: Mangiferin decreases plasma free fatty acids through promoting its catabolism in liver by activation of AMPK. PLoS One 7: e30782, 2015

20. Kevil CG and Bullard DC: In vitro culture and characterization of gene targeted mouse endothelium. Acta Physiol Scand 173: $151-157,2001$
21. Zhao Q, Sun Y, Ji Y, Xu L, Liu K, Liu B and Huang F: Total polyphenol of Anemarrhena asphodeloides ameliorates advanced glycation end products-induced endothelial dysfunction by regulation of AMP-Kinase. J Diabetes 6: 304-315, 2014.

22. Fan DM, Yang X, Huang LM, Ouyang GJ, Yang XX and Li M: Simultaneous detection of target CNVs and SNVs of thalassemia by multiplex PCR and next-generation sequencing. Mol Med Rep: Jan 24, 2019 (Epub ahead of print) doi: $10.3892 / \mathrm{mmr} .2019 .9896$.

23. Livak KJ and Schmittgen TD: Analysis of relative gene expression data using real-time quantitative PCR and the 2(-Delta Delta $\mathrm{C}(\mathrm{T})$ ) method. Methods 25: 402-408, 2001

24. Wang CN, Yang GH, Wang ZQ, Liu CW, Li TJ, Lai ZC, Miao SY, Wang LF and Liu B: Role of perivascular adipose tissue in nicotine-induced endothelial cell inflammatory responses. Mol Med Rep 14: 5713-5718, 2016.

25. Horimatsu T, Patel AS, Prasad R, Reid LE, Benson TW,Zarzour A, Ogbi M, Bruder do Nascimento T, Belin de Chantemele E, Stansfield BK, et al: Remote effects of transplanted prevascular adipose tissue on endothelial function and atherosclerosis. Cardiovasc Drug Ther 32: 503-510, 2018.

26. Xu X, Chen Y, Song J, Hou F, Ma X, Liu B and Huang F: Mangiferin suppresses endoplasmic reticulum stress in perivascular adipose tissue and prevents insulin resistance in the endothelium. Eur J Nutr 57: 1563-1575, 2018.

27. Larsson S and Voss U: Neuroprotective effects of vitamin D on high fat diet- and palmitic acid-induced enteric neuronal loss in mice. BMC Gastroenterol 18: 175, 2018

28. Ma Y, Li L, Shao Y, Bai X, Bai T and Huang X: Methotrexate improves perivascular adipose tissue/endothelial dysfunction via activation of AMPK/eNOS pathway. Mol Med Rep 15: 2353-2359, 2017.

29. Saez T, Toledo F and Sobrevia L: Extracellular vesicles and insulin resistance: A potential interaction in vascular dysfunction. Curr Vasc Pharmacol: Oct 1, 2018 (Epub ahead of print) doi: 10.2174/1570161116666181002095745.

30. Sheldon H, Heikamp E, Turley H, Dragovic R, Thomas $P$, Oon CE, Leek R, Edelmann M, Kessler B, Sainson RC, et al: New mechanism for Notch signaling to endothelium at a distance by Delta-like 4 incorporation into exosomes. Blood 116: 2385-2394, 2010.

31. Zhao W, Wang M, Shao L, Liao M, Liu K, Huang F and Liu B: The total phenolic fraction of Anemarrhena asphodeloides inhibits inflammation and reduces insulin resistance in adipocytes via regulation of AMP-kinase activity. Planta Med 80: 146-152, 2014

32. Lu Y, Chen Y, Li R, Liu Q, Wang N, Zhang Y, Li B and Fang Z: Protective effects of Danzhi jiangtang capsule on vascular endothelial damages induced by high-fat diet and palmitic acid. Biomed Pharmacother 107: 1631-1640, 2018.

33. Xu JZ, Chai YL and Zhang YL: Effect of rosuvastatin on high glucose-induced endoplasmic reticulum stress in human umbilical vein endothelial cells. Genet Mol Res: Oct 17, 2016 (Epub ahead of print). doi: 10.4238/gmr15048935.

34. Souza LE, Beckenkamp LR, Sobral LM, Fantacini DM, Melo FU, Borges JS, Leopoldino AM, Kashima S and Covas DT: Pre-culture in endothelial growth medium enhances the angiogenic properties of adipose-derived stem/stromal cells. Angiogenesis 21: 15-22, 2018.

35. Sun Y, Li J, Xiao N, Wang M, Kou J, Qi L, Huang F, Liu B and Liu K: Pharmacological activation of AMPK ameliorates perivascular adipose/endothelial dysfunction in a manner interdependent on AMPK and SIRT1. Pharmacol Res 89: 19-28, 2014.

36. Leng B, Tang F, Lu M, Zhang Z, Wang $\mathrm{H}$ and Zhang $\mathrm{Y}$ : Astragaloside IV improves vascular endothelial dysfunction by

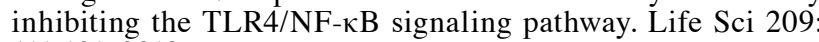
111-121, 2018.

This work is licensed under a Creative Commons Attribution 4.0 International (CC BY-NC 4.0) License 